\title{
The Cosmic Ray's Chaotic Motion in the Galaxy
}

\author{
Soji Ohara ${ }^{1}$, Takeharu Konishi ${ }^{1}$, Atsushi Mukai ${ }^{1}$ and Atsushi Iyono ${ }^{2}$ \\ 1. Department of Informatics, Naragakuen University, Ikomagun-Nara 6368503, Japan \\ 2. Department of Applied Science, Okayama University of Science, Okayama 7000005, Japan
}

\begin{abstract}
The time series data of ASS (air shower size) obtained by Nishimura-Kamata-Greisen formula are analyzed by the correlation dimension analysis to pick up the chaotic time series events. The arrival directions of the events in the chaotic time series are confirmed to have the anisotropy around 5, 15 and $20 \mathrm{~h}$ of R.A. (right ascension). In these directions, there are SNR (supernova remnants), AGN (active galactic nuclei) or $\mathrm{TeV}$ (tera electron volts) gamma ray sources. However, they are not expected to be the candidate of the chaotic cosmic rays which have the primary energy between $10^{13} \mathrm{eV}$ and $10^{16} \mathrm{eV}$, because the proton cosmic rays should migrate with Larmor rotation at radius $0.3 \mathrm{pc}$ (parsec) and the $\mathrm{TeV}$ gamma rays seem not to be emitted so often. Recently, Machida et al. reported the numerical results indicated that the magnetic fields were amplified on the azimuthal direction by the magneto rotational instability. The $20 \mathrm{~h}$ R.A is the azimuthal direction, while 5 and $15 \mathrm{~h} \mathrm{R}$.A. are around the direction perpendicular to the azimuthal direction. This amplified magnetic field could be the origin of the chaotic cosmic rays. The possible correlation between cosmic ray protons and the dark-matter is guessed also.
\end{abstract}

Key words: Anisotropy, chaotic cosmic rays, correlation dimension.

\section{Introduction}

The time series data of the air shower arrival time interval have been regarded as the random sequence without any correlation between them. This interpretation is reasonable under the recognition that the proton cosmic rays with the energy less than around $10^{15} \mathrm{eV}$ mix enough randomly through the itinerant motion in the Galactic magnetic field for long years. However, the chaotic feature and its anisotropy were found in the time series data of the arrival time interval of air showers [1-3]. The fractal wave model was proposed to insist that the chaotic cosmic rays arrive at the earth, forming wave trains like a fractal pattern which covers a large area on the earth. However, this model is not appropriate to the itinerant proton cosmic rays which have the kinetic energy around $10^{15} \mathrm{eV}$ because of random Larmor rotation with $r L=0.3 p c$ by the galactic magnetic field. The energy time series of protons which are accelerated by non-linear dynamics in SNR are assumed to have the chaotic feature, but it could not be expected that they arrive to the earth

Corresponding author: Soji Ohara, doctor, main research field: informatics. E-mail: ohara@naragakuen-u.jp. keeping the non-linear correlation through traveling several thousand light year distances. TeV gamma rays which are not influenced by the Galactic magnetic field are the second candidate of the chaotic cosmic rays. However, the results of recent observations by the telescope arrays indicate that gamma rays contribute less than $0.1 \%$ in all observed cosmic rays with the energy range between $10^{13} \mathrm{eV}$ and $10^{15} \mathrm{eV}$. Then, TeV gamma ray events, even though they have some chaotic correlation with each other, could not cause the chaotic feature of time series data of cosmic rays on our analysis. Then, what is the origin of the chaos of the time series data of cosmic rays?

\section{Materials}

\subsection{Chaos Analysis of the Time Series Data of ASS}

In this report, the object of analysis is the time series data of ASS rather than the arrival time interval in order to reach the information about the dynamics of the acceleration of cosmic rays. The ASS is calculated by Nishimura-Kamata-Greisen formula [4] $f(x)=c(s) x^{s-2}(x+1)^{s-4.5}$. In this formula, $f(x)$ is the lateral distribution function of electrons in air shower, $s$ is the 
age parameter and $x$ is equivalent to the distance between the center of air shower and the scintillation counter. The air shower data observed at three stations, Nara (N:34.60, E:135.68), Osaka (N:34.65, E:135.59), Okayama (N:34.69, E:133.92), from 2003 to 2013 are analyzed in this report. Nara station is located about $11 \mathrm{~km}$ southeast and Okayama station is located at 153 $\mathrm{km}$ west from Osaka station. The air shower array in each station consists of 5-7 plastic scintillation counters $\left(50 \times 50 \times 5 \mathrm{~cm}^{3}\right)$. The arrival times are recorded with a time resolution of $10 \mathrm{~ms}$ using the GPS (global position system). The arrival direction of the air shower is calculated with an arrival time difference between each counter obtained by a TDC (time to digital converter) on the assumption of a plane air shower front.

Fig. 1 shows the primary energy distribution of continuous 3,000 air showers observed at Osaka station in 2005 .

The correlation dimension analysis is executed with Grassberger-Procaccia method [5, 6] for every 200 or 150 continuous events whose duration is about $10 \mathrm{~h}$, shifting the first event by 2 events for each analysis. Fig. 2 shows a diagram of the analysis of the chaotic 200 time series data of ASS which is embedded in 11 dimensional phase space. $D_{m}$ value is defined by $d \ln C_{m} / d \ln r$, where $C_{m}$ is the number of the pair of vector point embedded in each 11 continuous time series data and $r$ is the pair distance. In the case of chaotic time series, $D_{m}$ has the constant value $D$ (correlation dimension) in some finite range of $r$ as shown in the formula $C_{m} \propto r^{D}$.

Using the embedding dimension $m=11$ in accordance with Takens's criterion [6] $\left(m>2 D_{\max }+1\right)$, the correlation dimension less than 5 could be detected.

The parallel slit bars around the plateau indicate the fixed area to get the graphical index of the flatness of the plateau of $D_{m}$ curve.

In the case of random noise the plateau disappears on the $D_{m}$ curve. If the sequence of the chaotic time series

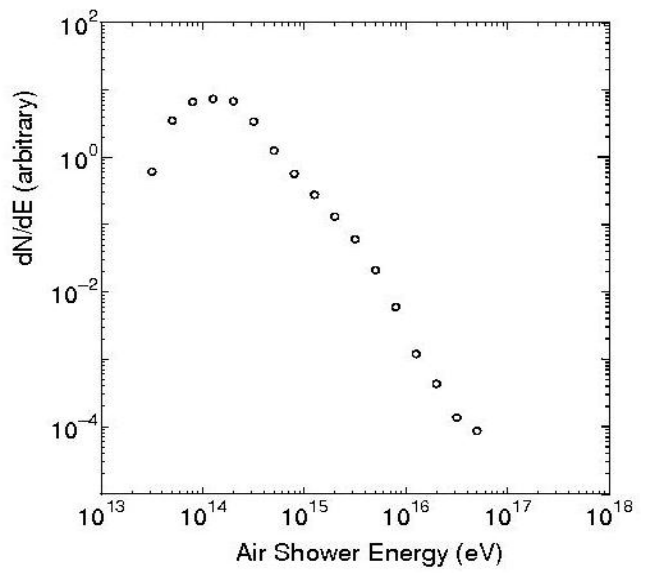

Fig. 1 The primary energy distribution of continuous 3,000 air showers observed at Osaka station in 2005.

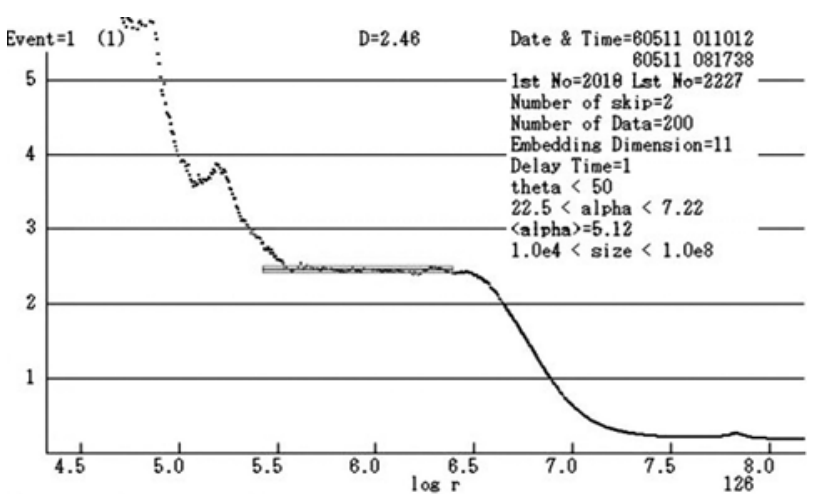

Fig. 2 The example of the diagram ( $D_{m}$ curve) of the correlation dimension analysis for the 200 chaotic time series of ASS observed at Osaka station in May 2006. The vertical position of the flatness indicates the correlation dimension.

data is randomized, then the plateau of $D_{m}$ disappears, so they are considered to be more chaotic rather than colored noise.

About 1.9 million time series data of ASS observed at 3 stations during 7 years are analyzed by the correlation dimension analysis.

\subsection{The Chaotic Cosmic Rays and Their Anisotropy}

Table 1 shows the average correlation dimension of groups of the chaotic time series data of ASS detected per year, total number of observed events, the number of detected chaotic groups and the relative frequency of chaotic groups for three stations. The chaotic groups which are joined to each other continuously are reckoned one as the number of chaotic group. The 
relative frequency of the chaotic group is significantly larger than the chance probability 0.001 . The range of correlation dimension is from 1.2 to 2.9 . The correlation dimension is so low that the non-linear dynamics which generates the chaotic feature of the time series data of ASS seems to be not so complicated as many factors are concerning with it.

The simultaneous detection rate of the chaotic time series data of ASS between two stations is not significant in this case, in contrast to the time series data of arrival time interval [3]. However the anisotropy and the periodicity of the chaotic feature, as mentioned below, indicate that the chaos of the time series data of ASS occurs as usual.

The anisotropy of the chaotic time series data of ASS is obtained by integrated R.A. data of the chaotic groups for each year, classifying the groups into three sections of the correlation dimension, $D \leqq 1.5,1.6 \leqq$ $D \leqq 2.0$ and $2.0<D$. The anisotropy is decided from the distribution of R.A. of the chaotic time series events in each section.

For example, Fig. 3 shows the R.A. distribution of the chaotic time series data of ASS for total 1,354 events obtained by integrating the groups which have the dimension less than equal 1.5, observed at Osaka station in 2006. The anisotropy of R.A. is detected by the weighted average for the frequency data around the peak. The error to detect the anisotropy of R.A. is plus or minus $1 \mathrm{~h}$. The anisotropies of R.A in Fig. 3 are $5 \mathrm{~h}$ and $18 \mathrm{~h}$ for instance. The anisotropy of the declination (Dec.) is detected by the weighted average of the Dec. distribution of the events which have the R.A. within the range plus or minus $2 \mathrm{~h}$ around the peak value of R.A. The error is plus or minus $2 \mathrm{deg}$. Table 2 shows the results of detected anisotropy of R.A. and corresponding Dec. of the chaotic air shower events classified by three sections of dimension. Obviously,

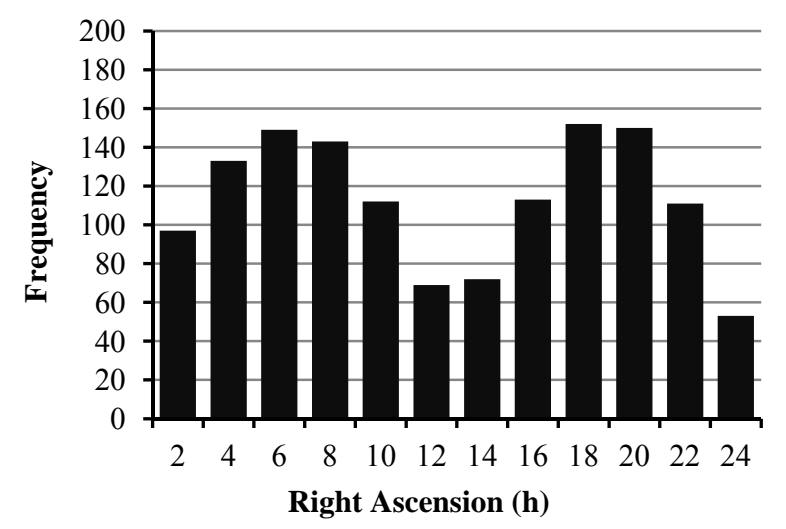

Fig. 3 The R.A. distribution of the chaotic time series data of ASS including 1,354 events of the chaotic groups which have the dimension less than equal 1.5, observed at Osaka station in 2006. It has two peaks at $5 \mathrm{~h}$ and $18 \mathrm{~h}$ (R.A.).

Table 1 Average correlation dimension of groups of chaotic time series detected per year for three stations. The total number of air shower events, the number of chaotic time series groups ASS and the relative frequency of the appearance of the chaotic group are also shown for each year at each station.

\begin{tabular}{llllll}
\hline Station & Year & $\begin{array}{l}\text { Number of } \\
\text { events }\end{array}$ & $\begin{array}{l}\text { Number of } \\
\text { chaos groups }\end{array}$ & $\begin{array}{l}\text { Relative } \\
\text { frequency }\end{array}$ & $\begin{array}{l}\text { Average } \\
\text { dimension }\end{array}$ \\
\hline Nara & 2003 & 134,330 & 24 & 0.036 & 1.6 \\
Nara & 2004 & 170,243 & 45 & 0.053 & 2.0 \\
Nara & 2005 & 220,038 & 42 & 0.038 & 2.1 \\
Nara & 2006 & 133,881 & 23 & 0.034 & 2.0 \\
Nara & 2007 & 77,398 & 8 & 0.016 & 1.8 \\
Nara & 2012 & 56,458 & 14 & 0.038 & 1.8 \\
Nara & 2013 & 91,078 & 16 & 0.026 & 1.7 \\
Osaka & 2003 & 114,986 & 37 & 0.064 & 1.8 \\
Osaka & 2004 & 145,015 & 25 & 0.031 & 1.9 \\
Osaka & 2005 & 128,914 & 14 & 0.020 & 2.0 \\
Osaka & 2006 & 155,792 & 16 & 0.018 & 1.7 \\
Osaka & 2007 & 79,569 & 26 & 0.065 & 1.8 \\
Okayama & 2004 & 111,450 & 13 & 0.018 & 2.0 \\
Okayama & 2005 & 134,653 & 23 & 0.029 & 1.9 \\
Okayama & 2006 & 143,206 & 24 & 0.025 & \\
\hline
\end{tabular}


Table 2 The anisotropy of the R.A. and the corresponding Dec. of the chaotic air shower time series detected for 1 year sectioned of three classes of correlation dimension. The hyphen in the cell means the period when the anisotropy is not clear. Double entry in a cell means two peaks of the anisotropy are found like Fig. 3.

\begin{tabular}{|c|c|c|c|c|}
\hline \multirow{2}{*}{ Station } & \multirow{2}{*}{ Year } & \multirow{2}{*}{$\begin{array}{l}\text { Dimension } \\
\text { range }\end{array}$} & \multicolumn{2}{|c|}{ Astronimical coordinates } \\
\hline & & & R.A.(h) & Dec(deg) \\
\hline Nara & 2003 & $1.2-1.5$ & 14 & 33 \\
\hline Nara & 2003 & $1.6-2.0$ & 22,5 & 35,34 \\
\hline Nara & 2004 & $1.6-2.0$ & 21 & 35 \\
\hline Nara & 2004 & $2.0+$ & 12 & 34 \\
\hline Nara & 2005 & $1.2-1.5$ & 15 & 31 \\
\hline Nara & 2005 & $1.6-2.0$ & 19 & 33 \\
\hline Nara & 2005 & $2.0+$ & 16 & 32 \\
\hline Nara & 2006 & $1.2-1.5$ & 22 & 35 \\
\hline Nara & 2006 & $1.6-2.0$ & 6,20 & 33,35 \\
\hline Nara & 2006 & $2.0+$ & 6 & 32 \\
\hline Nara & 2007 & $1.6-2.0$ & 6 & 33 \\
\hline Nara & 2012 & $1.2-1.5$ & 22 & 34 \\
\hline Nara & 2012 & $1.6-2.0$ & 20 & 36 \\
\hline Nara & 2013 & $1.2-1.5$ & 7 & 35 \\
\hline Nara & 2013 & $1.6-2.0$ & 7 & 33 \\
\hline Osaka & 2003 & $1.2-1.5$ & 6 & 34 \\
\hline Osaka & 2003 & $1.6-2.0$ & 15 & 35 \\
\hline Osaka & 2004 & $1.2-1.5$ & 22 & 34 \\
\hline Osaka & 2004 & $1.6-2.0$ & 6,18 & 32,32 \\
\hline Osaka & 2004 & $2.0+$ & 5,15 & 34,33 \\
\hline Osaka & 2005 & $1.2-1.5$ & 3,14 & 33,29 \\
\hline Osaka & 2005 & $1.6-2.0$ & 4,22 & 32,33 \\
\hline Osaka & 2005 & $2.0+$ & 2 & 32 \\
\hline Osaka & 2006 & $1.2-1.5$ & 5,18 & 30,30 \\
\hline Osaka & 2006 & $1.6-2.0$ & 4 & 35 \\
\hline Osaka & 2006 & $2.0+$ & 3 & 32 \\
\hline Osaka & 2007 & $1.2-1.5$ & 18 & 32 \\
\hline Osaka & 2007 & $1.6-2.0$ & 19 & 32 \\
\hline Osaka & 2007 & $2.0+$ & 21 & 33 \\
\hline Okayama & 2004 & $1.2-1.5$ & 7 & 33 \\
\hline Okayama & 2004 & $1.6-2.0$ & 6 & 33 \\
\hline Okayama & 2004 & $2.0+$ & 15 & 31 \\
\hline Okayama & 2005 & $1.2-1.5$ & 16 & 35 \\
\hline Okayama & 2005 & $2.0+$ & 15 & 33 \\
\hline Okayama & 2006 & $1.2-1.5$ & 6 & 37 \\
\hline Okayama & 2006 & $1.6-2.0$ & 22 & 34 \\
\hline Okayama & 2006 & $2.0+$ & 15 & 37 \\
\hline
\end{tabular}

the anisotropy value of R.A. deflects to three directions $5 \mathrm{~h}, 15 \mathrm{~h}$ and $20 \mathrm{~h}$.

The correlation dimension dependency of the anisotropy of the R.A. of the chaotic time series data of ASS is not obvious in Table 2.

The frequency of each anisotropy direction on R.A. is counted through the 7 years at 3 stations, dividing by the dimension 2.0 which is the mean value of the detected dimensions. The result is shown in Table 3 .

\subsection{The Periodic Change of the Chaotic Feature of the} Time Series Data of ASS

The chaotic feature of the time series data of ASS appears obviously in the particular group of events as mentioned above, but also appears always more or less 
Table 3 The correlation dimension dependency of the anisotropy of R.A. of the chaotic time series data of ASS. The numbers indicate the integrated number of the cases in Table 2.

\begin{tabular}{llll}
\hline Dimension & R.A. & & \\
\cline { 2 - 4 } range & $5 \pm 2 \mathrm{~h}$ & $15 \pm 2 \mathrm{~h}$ & $20 \pm 2 \mathrm{~h}$ \\
\hline $1.2-2.0$ & 14 & 5 & 14 \\
$2.0+$ & 4 & 6 & 1 \\
\hline
\end{tabular}

and the degree of the chaotic feature changes periodically with the time elapsing. Fig. 5 shows the change of the graphical index of the flatness of $D_{m}$ curve at the plateau area of $\ln r$ for each 200 time series data of ASS during a week observed at Osaka station in February 2005. The thick black line is the result of smoothing data processed by the moving average filter of window length $10 \mathrm{~h}$. It could be expected to have the period around 1.0 days. The FFT (fast fourier transform) analysis is executed for the time series data of the graphical index of the flatness of $D_{m}$ curve after the correlation dimension analysis for the original 2,048 time series data of ASS observed during a round 20 days of every month. The example of the result of the FFT analysis for the data which include the data in Fig. 5 is shown in Fig. 6. It obviously indicates the main period is 1.0 days.

\section{Results and Discussion}

Fig. 4 shows the scatter diagram of R.A. and Dec. for the corresponding data in Table 2. The distribution of the R.A. seems to be divided into three clusters. The average values of the R.A. of three clusters are $5 \mathrm{~h}, 15$ $\mathrm{h}$ and $20 \mathrm{~h}$. The solid circles indicate the anisotropy of the chaotic time series of ASS which have correlation dimension larger than 2.0.

For 70 groups of 2,048 events per month during 3 years, from 2004 to 2006, observed at 3 stations are analyzed using FFT. The frequency distribution of the main period detected by the FFT analysis is shown in Fig. 7 indicates 1.0 days is the general period of the chaotic feature of time series data of ASS.

The origin of the ghost peaks at 0.8 days or 1.3 days is confirmed by the FFT analysis for artificial time series sawtooth wave data whose period is 1.0 days

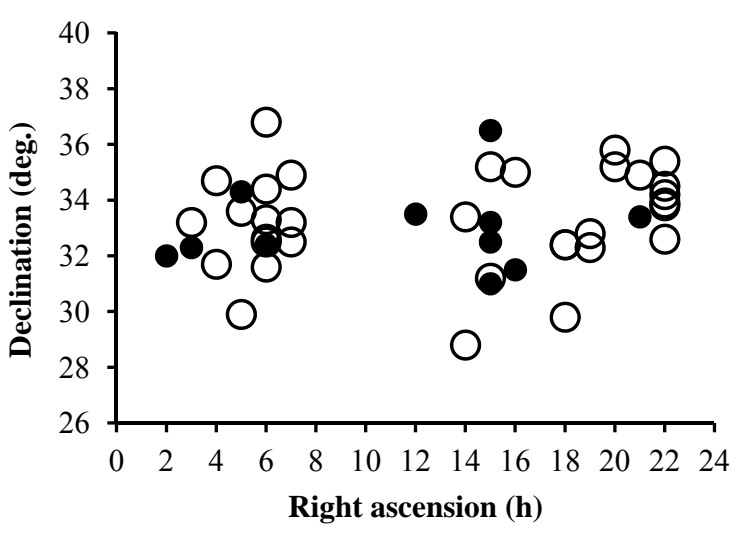

Fig. 4 The scatter diagram of R.A. and Dec. pairs of the chaotic time series of ASS groups shown in Table 2. Each circle represents more than 5 groups of the chaotic time series of ASS. In particular, the solid circles indicate the anisotropy of the chaotic time series of ASS which have correlation dimension larger than 2.0 .

including the phase inversion as shown in Fig. 8. In the case of the sine wave, such ghost peaks does not appear. The phase inversion may be happened with the arriving of the chaotic cosmic rays from another direction.

The period 1.0 days of the chaotic feature means that the origin of the chaos concerns to the particular arriving directions of cosmic rays, since the earth rotates with 1.0 day period in the space.

Then the chaotic cosmic rays have been confirmed to have the anisotropy of arrival direction of R.A. at around $5 \mathrm{~h}, 15 \mathrm{~h}$ and $20 \mathrm{~h}$ through the correlation dimension analysis and FFT analysis. The directions 5 $\mathrm{h}$ and $20 \mathrm{~h}$ of R.A. are the direction of the Galactic plane.

The main primary energy of the observed cosmic rays in this report is between $10^{13} \mathrm{eV}$ and $10^{16} \mathrm{eV}$. The proton cosmic rays which have the energy around $10^{15}$ $\mathrm{eV}$ rotates with Larmor radius around $0.3 \mathrm{pc}$. There has been dilemma about comprehending the origin of the chaotic cosmic rays. Although the chaotic feature of 


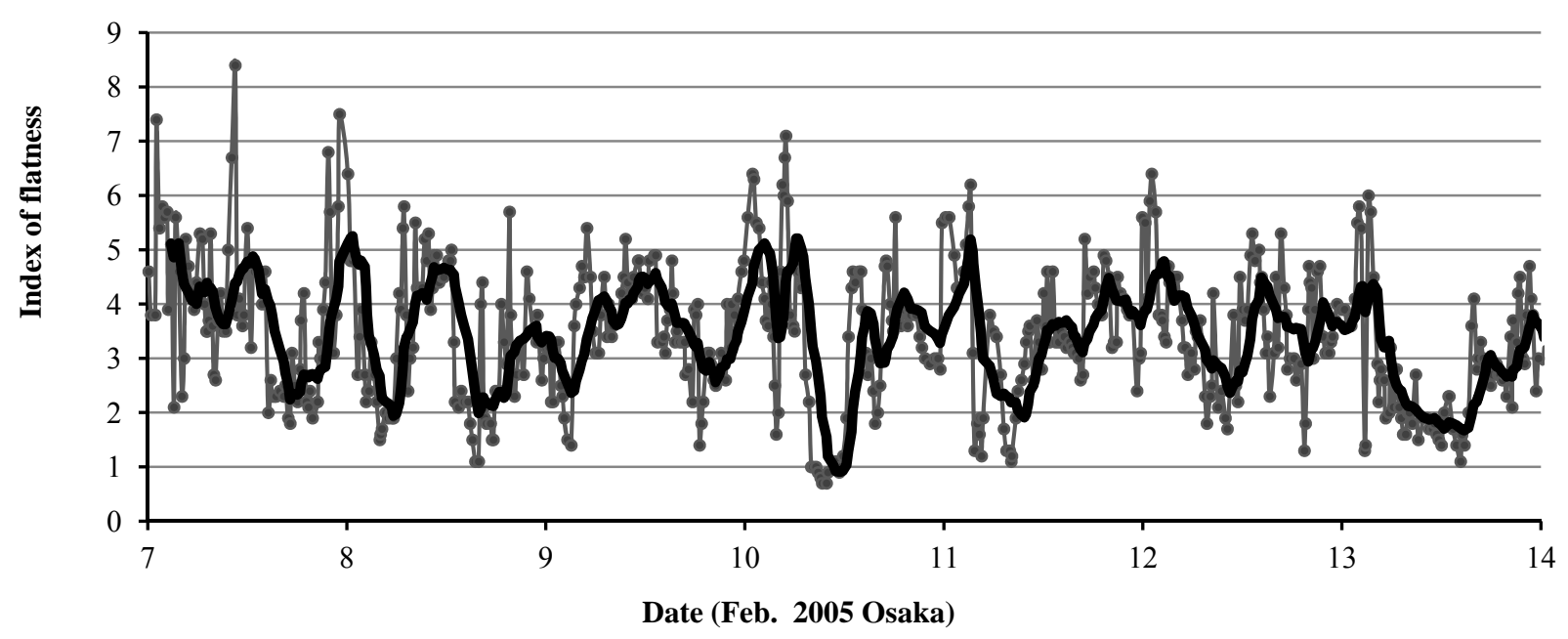

Fig. 5 The time variation of the chaotic feature of $\mathbf{2 0 0}$ time series of air shower size during a week observed at Osaka station. The vertical axis is the value of graphical index of the flatness of $D_{m}$ curve (plateau). The thick black line is the result of a moving average of the original result (the gray line).

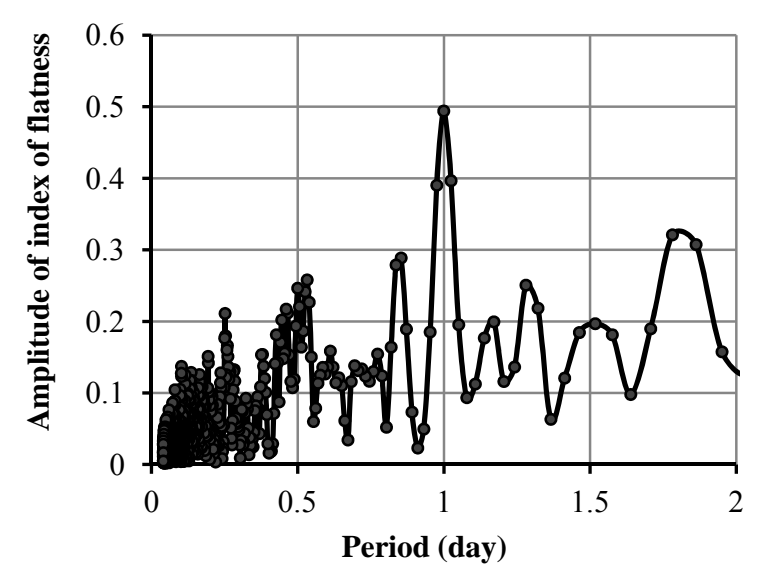

Fig. 6 The result of FFT analysis of 2048 data (during 20 days) which include the data in Fig. 5. The main peak appears at 1.0 day period.

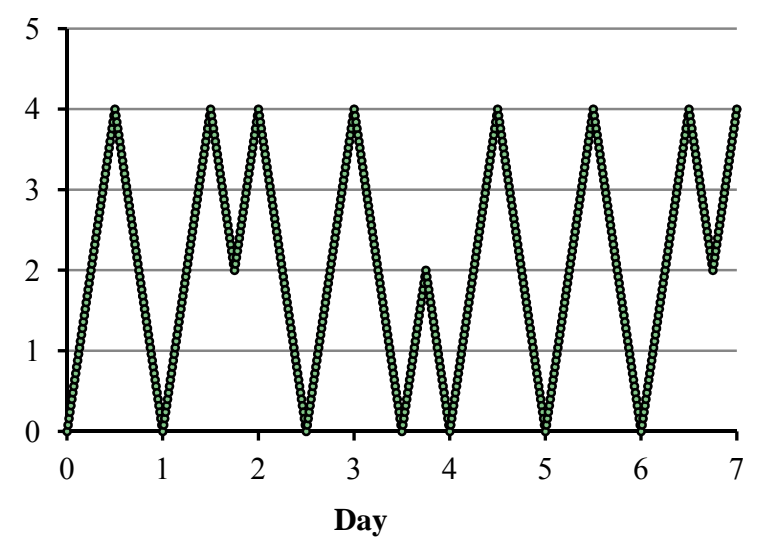

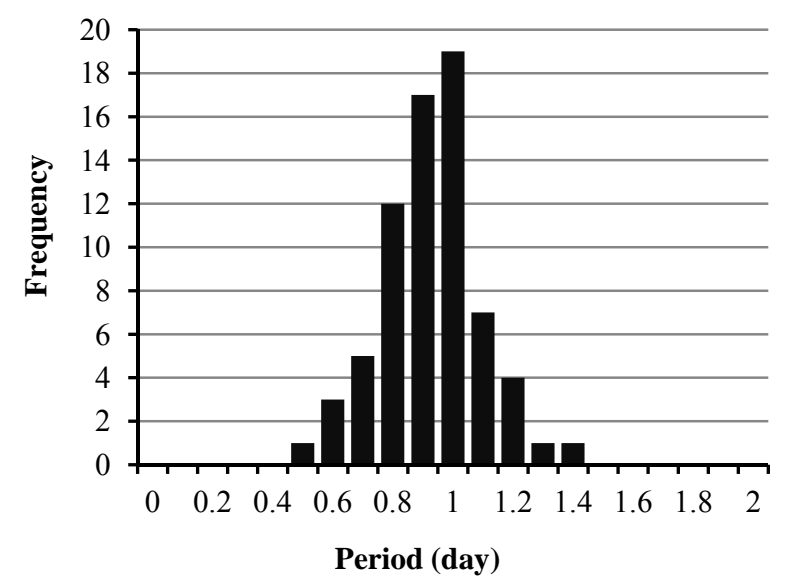

Fig. 7 The frequency distribution of the main period of the cosmic ray chaotic feature detected by FFT analysis. It shows obviously $\mathbf{1 . 0}$ day period is generally for the chaotic future.

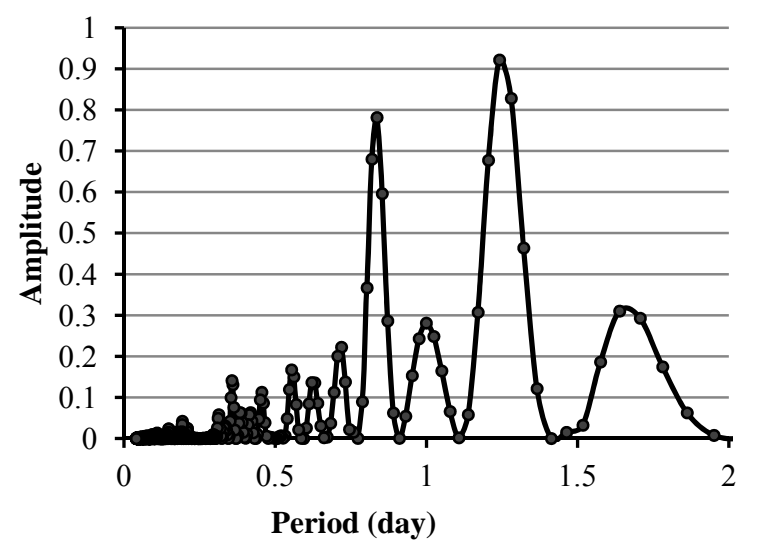

Fig. 8 Artificial sawtooth wave whose period is 1.0 days with phase inversion (left) and the result of FFT analysis which shows the ghost peaks (0.8 days and 1.3 days) of true peak (1.0 days) of period (right). 
the time series data of ASS means that the cosmic rays come from some celestial bodies through the identical non-linear dynamics. However, it could not be expected that any SNR or AGN accelerates the proton cosmic rays at the distance less than several pc. The TeV gamma ray sources, CygnusX-1 for examples, have been expected to be the source of the chaotic cosmic rays, because the $\mathrm{TeV}$ gamma-rays should not be influenced by the Galactic magnetic field, however, it is not reasonable to impose the chaotic feature on gamma-rays because the contribution ratio of gamma-rays to total cosmic rays with the energy between $10^{13} \mathrm{eV}$ and $10^{15} \mathrm{eV}$ is assumed to be around 0.1 percent. For the appearance of the chaotic feature on the correlation dimension analysis, more than $30 \%$ contribution of the chaotic events seems to be necessary at least.

Then, it is difficult to identify a particular SNR, ANG or another TeV gamma ray source as the source of the chaotic time series data of ASS in spite of that the systematic classification of the anisotropy of the chaotic feature shown in Fig. 4 and the evidence of usual arriving of the chaotic cosmic rays with 1.0 day period as shown in Fig. 7.

The new idea about the origin of the chaotic cosmic rays has been hinted from the recent theoretical simulation reported by Machida et al. [7]. They have reported the results of three-dimensional magneto hydrodynamic simulations of dynamo activities in galactic gaseous disks. Their numerical results indicate the growth of azimuthal magnetic fields non-symmetric to the equatorial plane. It could be supposed that the itinerant protons as cosmic rays are influenced by the amplified azimuthal magnetic field in the Galaxy for long times and the chaotic feature could be obtained so that the chaos of time series data of ASS have anisotropy of arrival direction around the direction of Galaxy plane.

Table 3 shows the chaotic time series of ASS which have the anisotropy of $5 \mathrm{~h}$ and $20 \mathrm{~h}$ R.A. tend to give the low correlation dimension. This result indicates that the dynamic process of the acceleration of cosmic rays on the galactic plane of the amplified magnetic field may be not so complicated, the number of factors concerning the process is up to 2 . On the other hand, the chaotic time series of ASS which have the anisotropy of $15 \mathrm{~h}$ R.A. do not depend on the correlation dimension. A little complex structure of magnetic field may concern to the acceleration of cosmic rays arriving from this direction.

The average R.A. and Dec. of three clusters shown in Fig. 4 are ( $\alpha: 5$ h, $\delta: 33 \mathrm{deg}),(\alpha: 15 \mathrm{~h}, \delta: 33 \mathrm{deg})$ and $(\alpha: 20$ h, $\delta: 34 \mathrm{deg})$ which are $(1: 170.5, \mathrm{~b}:-6.6),(1: 52.9, \mathrm{~b}: 62.7)$ and (1:70.0, b:3.0) . Fig. 9 illustrates these directions with the direction of the amplified magnetic fluxes reported by $\mathrm{M}$. Machida et al..

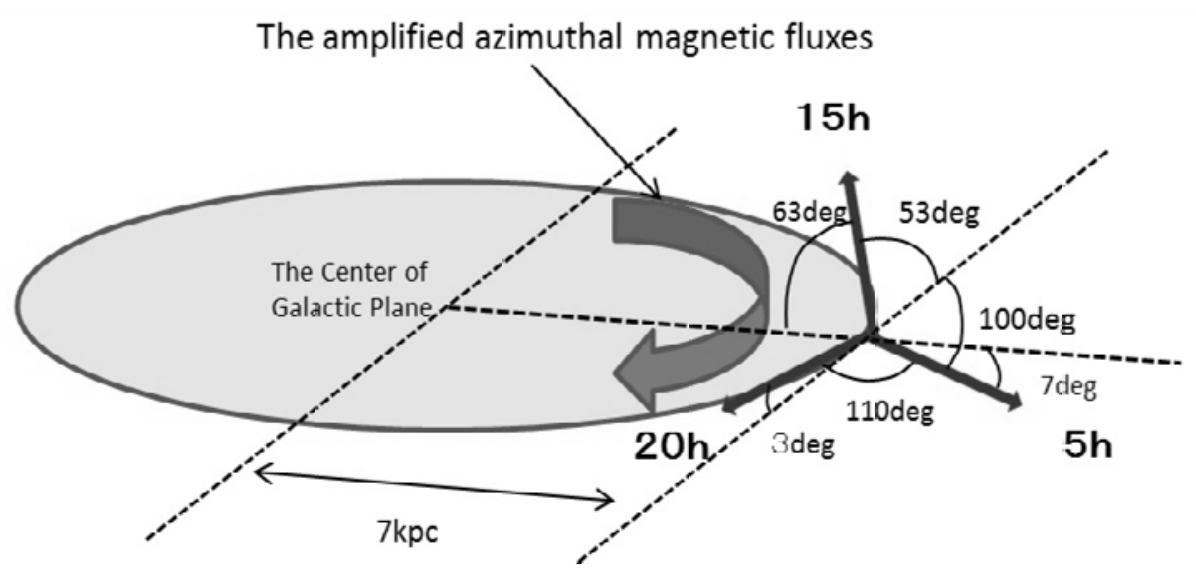

Fig. 9 The illustration of the three anisotropies of the chaotic time series data of ASS together with the amplified azimuthal magnetic fluxes reported by M.Machida et al. The chaotic feature of the cosmic rays is considered to be caused by the amplified azimuthal magnetic fluxes in the Galaxy. 
The anisotropy direction $20 \mathrm{~h}$ (R.A.) is the direction of CygnusX-1 and the direction of WIMP (weakly interacting massive particle) wind. The frequency of the chaotic time series of ASS observed during October to March is 6 times of the frequency of the chaotic time series of ASS observed during April to September in 2004 to 2006 in the case of that the anisotropy of R.A. is $20 \mathrm{~h}$. For the anisotropy of $5 \mathrm{~h}$ and $15 \mathrm{~h}$, the frequency is comparable between summer season and winter season. This result may mean that WIMP wind from the direction of CygnusX-1 which would be strong in summer season [8] disturb the appearance of chaotic feature of the time series data of the proton cosmic rays by some correlations.

\section{Conclusion}

The anisotropy of the chaotic cosmic rays could be considered to concern well with the amplified azimuthal magnetic fields in the Galaxy simulated by M. Machida et al. [7]. Though the detail mechanism should be studied hereafter. The chaotic time series events should arrive at the earth after traveling together with several Larmor rotations under the same field of the amplified magnetic fluxes. Moreover, this approach with the correlation dimension analysis of the time series data of ASS may give some information about the dark matter in the Galaxy.

\section{References}

[1] Ohara, S., Kitamura, T., Chikawa, M., Unno, W., Tsuji, K., Konishi, T., and Masaki, I. 1994. "Chaotic Feature in Cosmic Ray Air Showers with Energies Larger than $3 \times$ $10^{14}$ eV." Proc. of 8th Int. Symposium in Very High Energy Cosmic Ray Interactions, Tokyo (July) 628-67.

[2] Ohara, S., Konishi, T., and LAAS group. 2003. "Chaos in Different Far-off Cosmic Rays: A Fractal Wave Model.” Journal of Physics G: Nuclear and Particle Physics 29: 2065-77.

[3] Ohara, S., Konishi, T., Mukai, A., Iyono, A., and LAAS group. 2011. "The Anisotropy of Cosmic Ray Pursued with Chaos Analysis." Proc. 32nd ICRC, Beijing.

[4] Kamata, K., and Nishimura, J. 1958. "The Lateral and the Angular Structure Functions of Electron Showers." Progress of Theoretical Physics Supplement 6: 93-155.

[5] Grassberger, P., and Procaccia, I. 1983. "Characterization of Strange Attractor.” Physical Review Letters, 346-9.

[6] Takens, F. 1981. "Detecting Strange Attractors in Turbulence." Lecture Notes in Mathematics, Springer, 366-80.

[7] Machida, M., Nakamura, K., Kudoh, T., Akahori, T., Sofue, Y., and Matsumoto, R. 2013. "Dynamo Activities Driven by Magnetorotational Instability and the Parker Instability in Galactic Gaseous Disks." Astrophysical Jounal 10 (February): 1-9.

[8] Caldwell, R., and Kamionkowski, M. 2009. "Dark Matter and Dark Energy.” Nature. 458 (2) (April): 587-9. 